21

22 *Corresponding author: email: tkaranf@clemson.edu; phone: +1-864-656-1005; fax: +1-864-656-0672

\section{Adsorption of Halogenated Aliphatic Contaminants by Graphene}

Nanomaterials

\author{
Yang Zhou, Onur Guven Apul, Tanju Karanfil* \\ Department of Environmental Engineering and Earth Sciences \\ Clemson University \\ 342 Computer Court \\ Anderson, SC 29625 \\ United States
} \section{4} 5

April 12, 2015

23 


\section{ABSTRACT}

25 In this study, adsorption of ten environmentally halogenated aliphatic synthetic organic 26 compounds (SOCs) by a pristine graphene nanosheet (GNS) and a reduced graphene oxide (rGO)

27 was examined, and their adsorption behaviors were compared with those of a single-walled 28 carbon nanotube (SWCNT) and a granular activated carbon (GAC). In addition, the impacts of 29 background water components (i.e., natural organic matter (NOM), ionic strength (IS) and $\mathrm{pH}$ ) on the SOC adsorption behavior were investigated. The results indicated HD3000 and SWCNT

31 with higher microporous volumes exhibited higher adsorption capacities for the selected 32 aliphatic SOCs than graphenes, demonstrating microporosity of carbonaceous adsorbents played 33 an important role in the adsorption. Analysis of adsorption isotherms demonstrated that 34 hydrophobic interactions were the dominant contributor to the adsorption of aliphatic SOCs by graphenes. However, $\pi-\pi$ electron donor-acceptor and van der Waals interactions are likely the

36 additional mechanisms contributing to the adsorption of aliphatic SOCs on graphenes. Among

37 the three background solution components examined, NOM showed the most influential effect on adsorption of the selected aliphatic SOCs, while $\mathrm{pH}$ and ionic strength had a negligible effects.

39 The NOM competition on aliphatic adsorption was less pronounced on graphenes than SWCNT.

40 Overall, in terms of adsorption capacities, graphenes tested in this study did not exhibit a major 41 advantage over SWCNT and GAC for the adsorption of aliphatic SOCs.

43 Keywords: Adsorption, Aliphatic Synthetic Organic Compounds, Graphenes, Carbon 44 Nanotubes, Activated Carbons, Natural Organic Matter 


\section{Introduction}

Graphenes are two-dimensional single layered, $\mathrm{sp}^{2}$ hybridized carbon atoms densely packed as a hexagonal honeycomb lattice and they can be visualized as basic building blocks for

49 fullerenes, carbon nanotubes (CNTs) and graphite [Novoselov et al., 2004]. The unique structure endows graphenes with outstanding mechanical, optical and electronic properties [Geim, 2009;

51 Lee et al., 2008; Novoselov et al., 2005], which make them ideal candidates for a wide range of 52 commercial applications. Commercial production and industrial scale application of graphenes 53 are expected to grow exponentially over the next decades [Geim and Novoselov, 2007; Li and 54 Kraner, 2008]. However, due to their mass production, the release of carbonaceous 55 nanomaterials (such as graphenes) into environment possess various health and environmental 56 risks for plants, animals and humans [Nowack et al. 2012; Upadhyayula et al. 2009; Yu et al. 57 2014; Zhang et al., 2010; Zhao et al., 2014]. Furthermore, some of the negative impacts might be 58 increased as a result of adsorbing synthetic organic compounds (SOCs) by these nanomaterials, and the fate and transport of SOCs in the environment can be altered.”.

Graphenes are hydrophobic nanomaterials with large specific surface areas (SSA); and 61 they have been evaluated as promising adsorbents to remove SOCs from water [Zhao et al et al., 62 2011; Ramesha et al., 2011; Ji et al., 2013; Sharma and Das, 2013; Zhao et al., 2011; Li et al., 63 2012; Gao et al., 2012; Li et al., 2013; Apul et al., 2013; Wu et al., 2011]. In our previous study 64 [Apul et al., 2013], graphenes were shown to have higher or comparable adsorption capacities to selected 65 carbon nanotubes and granular activated carbons. In addition, the impact of NOM competition on the 66 adsorption capacity of graphenes was less severe. Therefore, from an engineering perspective, graphenes 67 may serve as novel and alternative adsorbents in engineered treatment system in future [Yu et al. 2015]. 68 To date, the majority of the tested SOCs were aromatic compounds; and no study has examined 
69 the adsorption of aliphatics by graphenes. Several aliphatic SOCs are common organic pollutants

70 that were either regulated by United States Environmental Protection Agency (US EPA) under

71 Priority Pollutants List (e.g., trichloroethylene, tetrachloroethylene, 1,1-dichloroethylene) or

72 listed under Candidate Contaminant List 3 (CCL3) (e.g., 1,1,1,2-tetrachloroethane, 1,1-

73 dichloroethane). Given the paucity of data on this topic in literature, it is important to understand

74 adsorption of aliphatic SOCs by graphenes to adequately assess the environmental impact and

75 engineering applications of graphenes. Therefore, the main objectives of this study were to (i)

76 investigate the factors controlling adsorption of halogenated aliphatic SOCs by graphenes in

77 terms of adsorbent characteristics, adsorbate properties and background solution chemistry, and

78 to (ii) evaluate the application potential of graphenes as alternative adsorbents in treatment

79 systems by comparing their adsorption capacities with those of single-walled carbon nanotubes

80 (SWCNT) and granular activated carbon (GAC).

\section{2. Materials and methods}

\section{$83 \quad 2.1$. Adsorbents}

Four carbonaceous adsorbents: pristine graphene nanosheets (GNS, Angstron Materials

85 Inc.), reduced graphene oxide (rGO, Graphenea CO., Ltd.), SWCNT (Chengdu Organic

86 Chemcials Co., Ltd.) and GAC (HD3000, Norit Inc.) were used. GNS, rGO, and SWCNT were

87 used as received from the manufacturers, while HD3000 was ground to 200-325 $\mu$ m mesh size

88 prior to use. Selected physicochemical properties of the four carbonaceous adsorbents are 89 summarized in Table 1. 


\subsection{Adsorbates}

Ten aliphatic SOCs with different properties were purchased from Acros (PCE, > 99\%),

Fluka (12DCP, > 99\%; 12DBE, > 98\%), Matrix Scientific (DBCP, > 98\%), Alpha Easer (TCE, >

94 99.5\%), TCI (1112TeCA, > 99\%), Baker Analytical (111TCA, > 96.7\%) and Sigma Aldrich

95 (112TCA, > 96\%; 11DCE, > 99\%; $\left.\mathrm{CCl}_{4},>99.9 \%\right)$. Both of these aliphatic SOCs represented

96 common organic pollutants that were either listed under Priority Pollutants List or CCL3, and

97 they differ in molecular size, hydrophobicity, number of double bonds, and polarizability.

98 Therefore, they were employed as probe molecules to cover some typical adsorbate-adsorbent

99 interactions. Abbreviations, properties and molecular structures of the aliphatic SOCs are 100 summarized in Tables 2 and S1 in Supporting Information.

\subsection{NOM solution}

The natural organic matter (NOM) was isolated from the influent of Myrtle Beach 104 drinking water treatment plant in South Carolina using a reverse osmosis and followed by resin 105 fractionation, as described elsewhere [Song et al., 2009]. SUVA 254 , defined as the ratio of UV 106 absorbance at $254 \mathrm{~nm}$ divided by the dissolved organic carbon (DOC) concentration, is a 107 quantitative measurement of the aromatic content per unit concentration of organic carbon in 108 water [Karanfil et al., 2003]. Natural waters with high SUVA 254 values (e.g., more than 4.0 109 L/mg-m) have organic matter with relatively high contents of hydrophobic, aromatic, and high 110 molecular weight components, whereas waters with low SUVA 254 values (e.g., less than 2.0 111 L/mg-m) contain mostly non-humic, hydrophilic and low molecular weight material. The 
112 SUVA $_{254}$ value of the NOM solution used was around $4.0 \mathrm{~L} / \mathrm{mg}-\mathrm{m}$, indicating it was rich in 113 aromatic components.

\section{2.4. Characterization of adsorbents}

Several techniques were used for the characterization of carbonaceous adsorbents.

117 Nitrogen gas adsorption at $77 \mathrm{~K}$ was performed with a physisorption analyzer (Micromeritics 118 ASAP 2010) to determine the SSA, total pore volumes (PV) and pore size distributions (PSD) of 119 the four adsorbents. The Brunauer-Emmett-Teller (BET) equation was used to calculate SSA. 120 The total PV were obtained from the adsorbed volume of nitrogen near the saturation point $\left(\mathrm{P} / \mathrm{P}_{0}\right.$ $121=0.99)$. PSD of adsorbents were determined from the nitrogen isotherms using the Density 122 Functional Theory (DFT) model. Oxygen contents of the carbonaceous adsorbents were 123 measured using a Flash Elemental Analyzer 1112 series (Thermo Electron Corporation). In 124 addition, $\mathrm{pH}$ of the point of zero charge $\left(\mathrm{pH}_{\mathrm{PZC}}\right)$ of adsorbents was determined using $\mathrm{pH}$ 125 equilibration technique. The details about these characterization methods have been provided 126 elsewhere [Dastgheib et al., 2004].

\subsection{Adsorption experiments}

Constant carbon dose aqueous phase isotherm experiments were conducted using 130 completely mixed batch reactors (125 mL glass bottles with Teflon-lined screw caps). Two types 131 of isotherms were conducted at room temperature $\left(20 \pm 3{ }^{\circ} \mathrm{C}\right)$ :

(1) Distilled and deionized water (DDW) experiments: Concentrated stock solution of 
134 first filled with DDW and no headspace, and spiked with predetermined volumes of aliphatic 135 SOCs from their methanol stock solutions. For the graphene experiments, the bottles with 136 adsorbents were initially half filled with DDW, sonicated for 20 min, and completely filled with 137 DDW prior to spiking aliphatic SOCs. The volume percentage of the methanol spiked solution 138 per bottle was kept below $0.4 \%(\mathrm{v} / \mathrm{v})$ to minimize the co-solvent effect. Preliminary kinetic 139 experiments were preformed for TCE adsorption onto four carbonaceous adsorbents; and the 140 results showed that a time period of seven days was sufficient to reach equilibrium (Figure S1). 141 Thus, the bottles with no headspace were placed into a rotary tumbler for one week. The solution $142 \mathrm{pH}$ remained around 6.5 during the experiments. To investigate the effect of $\mathrm{pH}$ on adsorption, 143 additional adsorption isotherm experiments were conducted at $\mathrm{pH} 3$ and 11, where the solution $144 \mathrm{pH}$ was adjusted by $0.1 \mathrm{~mol} / \mathrm{L} \mathrm{HCl}$ and $\mathrm{NaOH}$. For ionic strength (IS) effect experiments, the 145 same experimental procedure was used, except the ionic strength was adjusted with $\mathrm{NaCl}$ to 146 three levels (IS=0.001, 0.01, $0.1 \mathrm{M}$ ). The ranges for $\mathrm{pH}$ and ionic strength were kept wider than 147 those typically used in water and wastewater treatment or found in natural water systems to 148 examine their impact in a wider parametric range.

(2) Preloading experiments: The NOM effect on adsorption of aliphatic SOCs by 150 different carbonaceous adsobents was examined under preloading condition, giving an advantage 151 to NOM adsorption prior to that of the SOCs, which represents the most severe NOM 152 competition condition. For the preloading experiments, bottles containing about $5 \mathrm{mg}$ adsorbents 153 were first filled fully with $3 \mathrm{mg}$ DOC/L NOM solution buffered with $1 \mathrm{mM}$ $154 \mathrm{NaH}_{2} \mathrm{PO}_{4} \cdot \mathrm{H}_{2} \mathrm{O} / \mathrm{Na}_{2} \mathrm{HPO}_{4} \cdot 7 \mathrm{H}_{2} \mathrm{O}$ and adjusted to $\mathrm{pH}$ 7.0, then the bottles with zero headspace were 155 placed into a rotary tumbler. After four days, predetermined volumes of aliphatic SOC stock 156 solutions were directly spiked into the bottles, and then the headspace-free bottles were tumbled 
157 again for an additional week. In NOM preloading experiments, $200 \mathrm{mg} / \mathrm{L} \mathrm{NaN}_{3}$ was added to 158 NOM solution to minimize any biological activity.

After the equilibrium period of isotherm experiments, bottles were placed on a bench 160 overnight to allow settling of the adsorbents, and supernatants of samples in the bottles were 161 transferred to $10 \mathrm{~mL}$ centrifuge tube for centrifugation to remove the remaining adsorbents. The 162 supernatants were extracted with hexane by liquid: liquid extraction and analyzed using a gas 163 chromatograph with a micro-electron capture detector (GC- $\mu$ ECD) equipped with a Rxi-624Sil 164 MS Column (Restek, USA). Bottles without any adsorbents served as blanks to monitor the loss 165 of adsorbates during the experiments, which were found to be negligible.

\section{$167 \quad$ 2.6. Isotherm modeling} (LFM) and Polanyi-Manes models (PMM), were employed to fit the experimental data. The modeling results showed that FM model resulted in good fits with meaningful parameter values

171 for every case (Tables S2 to S5). Therefore, the FM model was selected to fit the adsorption data 172 in this study:

$173 \quad \mathrm{q}_{\mathrm{e}}=\mathrm{K}_{\mathrm{F}} \mathrm{C}_{\mathrm{e}}^{\mathrm{n}}$

174 where $q_{e}$ and $C_{e}$ represent the solid-phase equilibrium concentration (mg/g) and the liquid-phase 175 equilibrium concentration $\left(\mu \mathrm{g} / \mathrm{L}\right.$ or $\mathrm{mg} / \mathrm{L}$ ), respectively, $\mathrm{K}_{\mathrm{F}}$ is the unit-capacity parameter $176\left((\mathrm{mg} / \mathrm{g}) / \mathrm{C}_{e}{ }^{\mathrm{n}}\right)$, equal to the amount adsorbed at a value of $\mathrm{C}_{e}$ equal to unity, and $\mathrm{n}$ is a 177 dimensionless parameter related to the surface heterogeneity. Two $\mathrm{K}_{\mathrm{F}}$ parameters $\left(\mathrm{K}_{\mathrm{F} \mu}\right.$ and $\left.\mathrm{K}_{\mathrm{Fm}}\right)$ 
178 were examined for SOCs adsorption capacities at equilibrium concentrations of $1 \mu \mathrm{g} / \mathrm{L}$ and $1791 \mathrm{mg} / \mathrm{L}$, respectively.

\section{Result and discussion}

\subsection{Adsorbent characterization}

The results of SSA, total PV, PSD, oxygen content and $\mathrm{pH}_{\mathrm{PZC}}$ of the four carbonaceous 184 adsorbents are summarized in Table 1. The SSA of GNS and rGO were 666 and $497 \mathrm{~m}^{2} / \mathrm{g}$, 185 respectively; and they were considerably smaller than the theoretically calculated SSA of single186 layered graphene nanomaterials $\left(2630 \mathrm{~m}^{2} / \mathrm{g}\right.$ ) [Stoller et al., 2008]. The much lower SSA 187 indicated that graphenes were not present as exposed single-sheets, instead they formed bundles 188 decreasing the SSA. Very high total PV $\left(>3 \mathrm{~cm}^{3} / \mathrm{g}\right)$ of GNS indicated aggregation may be 189 confining large amounts of space within its 'house-of-cards' like bundle structure. GNS had six 190 times higher total PV than rGO, indicating the oxygen content of rGO may be inhibiting the 191 formation of bulky pores (especially macropores) within its bundle structure. The lacking 192 macropores may also be attributed to tighter aggregation of rGO due to hydrogen bonding 193 between the oxygen containing functional groups within the graphene bundle. When comparing 194 the graphenes with SWCNT and HD3000, no major difference was observed in SSA, total PV 195 and PSD besides the notably large macropore volume of GNS. In addition, rGO had higher oxygen content (17.5\%) than other adsorbents as expected, which suggested that the surface of

197 rGO is more hydrophilic. The low $\mathrm{pH}_{\mathrm{PzC}}$ value of rGO indicated a net negative charge under 198 neutral conditions due to the presence of oxygen-containing functional groups. 


\subsection{The effect of adsorbent properties on adsorption of aliphatics}

201

202

221 2013].

Adsorption isotherms of PCE, TCE and 1,1-DCE by the four carbonaceous adsorbents in DDW are presented in Figure 1 and the Freundlich isotherm parameters were summarized in Table S6. Some major observations from the isotherms and isotherm parameters are as follow: HD3000 and SWCNT exhibited higher adsorption capacities than graphenes for all aliphatic SOCs tested. The adsorption isotherms were normalized according to the SSA and micropore volumes of adsorbents. However, after normalization, the adsorbed amount of aliphatic SOCs by HD3000 and SWCNT were still higher than those of GNS and rGO (Figures 1-D, E, F, G, H, I). Adsorbents with higher micropore volumes (HD3000 and SWCNT) showed higher adsorption capacities; however, SSA or micropore volume difference did not completely explain the adsorption capacity difference. Because, SSA, total PV and PSD parameters were obtained in the bulk phase of adsorbents via $\mathrm{N}_{2}$ adsorption, the aggregation of CNTs and graphenes in aqueous phase may change the availability of sorption sites for aliphatic SOCs unlike rigid activated carbon pore structure.

Pristine GNS exhibited higher uptake for the aliphatic SOCs than rGO even after surface area normalization, which was attributed to its low surface polarity. Oxygen-containing functional groups on carbonaceous adsorbents can decrease the accessibility of adsorbents for aliphatic SOCs either through (i) a decrease due to increasing hydrophilicity (i.e. polarity) of the surface, or (ii) a decrease in the number of available adsorption sites due to water cluster formation around the oxygen-containing functional groups. Similar effect of oxygen content of graphenes was also observed on the adsorption of aromatic organic contaminants [Apul et al., 


\subsection{The effect of aliphatic SOC properties on adsorption}

To investigate the effect of aliphatic SOC properties, adsorption isotherms of the

225 aliphatic SOCs on GNS and rGO are compared in Figures 2-A, B. Hydrophobic interactions are

226 important driving forces for adsorption of SOCs from aqueous solutions. To investigate the

227 contribution of hydrophobicity of the aliphatic SOCs on adsorption, the adsorption isotherms

228 were normalized according to their solubilities (Figures 2-C, D). After the solubility

229 normalization, the separation in the adsorption isotherms on both GNS and rGO were greatly

230 reduced but isotherms did not converge on a single line. This indicates that hydrophobicity of the

231 aliphatic SOCs was an important driving force for adsorption, and there were other factors

232 contributing to adsorption.

To further investigate the effect of the other SOC properties on adsorption, the PCE, TCE

234 and 11DCE isotherms were compared. These compounds have similar molecular structures and 235 configurations but different number of chlorine atoms. As shown in Figures 3-A, B, adsorption 236 affinities of the three aliphatic SOCs by GNS and rGO followed the same order: PCE > TCE > 237 11DCE. Solubility normalization greatly reduced the differences in the adsorption isotherms, 238 although isotherms did not converge on a single line (Figures 3-C, D), and still followed the 239 same order with smaller differences. The difference in the strengths of $\pi$ - $\pi$ electron donor240 acceptor (EDA) interactions of these three aliphatics with the graphenes might influence their 241 adsorption. The highly polarizable graphene sheet can play an amphoteric role attracting both $\pi$ 242 electron-acceptors and $\pi$ electron-donors to the surface; and chlorine atoms on carbon double 243 bonds of the three SOCs can serve as $\pi$ electron acceptors, which can involve in adsorption 244 interactions between the SOCs and graphenes through $\pi-\pi$ EDA complex formation. Therefore, 245 adsorption affinities of the three aliphatic SOCs by graphenes increase with increasing number of 
246 chlorine atoms. Another possibility influencing adsorption of these three compounds is the 247 nonspecific interactions generally referred to as van der Waals forces. These interactions can 248 exist between molecules regardless of their chemical structures; and they are proportional to the 249 product of polarizability or dipole moment of adsorbates and adsorbents. Thus, stronger van der 250 Waals interactions between PCE and graphenes might occur due to its higher polarizability. The 251 separation in solubility-normalized isotherms of the three aliphatic SOCs on both GNS and rGO 252 were further reduced after polarizability normalization (Figures 3-E, F). This additional 253 reduction suggested that increasing polarizability of aliphatic SOCs may also have positive 254 effects in their adsorption. To further examine the effect of polarizability, two isomer aliphatic 255 SOCs (111TCA and 112TCA) with different polarizability were selected. Figure 4 compares 256 their adsorption isotherms on GNS and rGO after solubility normalization to eliminate the 257 difference in the hydrophobicity of these two compounds. The results showed both GNS and 258 rGO showed higher adsorption capacities for 112TCA than 111TCA. As seen in Table 2, 259 112TCA has higher polarizability than 111TCA (0.68 vs. 0.41$)$; thus stronger van der Waals 260 interactions between 112TCA and graphenes may be expected.

Presence of $\pi$-electrons in the structure of aliphatic SOCs may be another factor affecting

262 their adsorption on graphenes. Aromatic SOCs have strong $\pi-\pi$ bonding interactions with 263 graphene surface due to presence of resonating $\pi$-electrons in their benzene rings [Apul et al., 264 2013]. To investigate whether the $\pi$-electrons of aliphatic SOCs exert the effect on their 265 adsorption, solubility-normalized adsorption isotherms of PCE vs. 1112TeCA and TCE vs. 266 112TCA were compared (Figure 5) because they have similar molecular structures but different 267 carbon bonds. After the solubility normalization, the separation between their adsorption 268 isotherms was reduced greatly; and the isotherms almost converged on a single line, which 
269 indicated the $\pi$-electrons of aliphatic SOCs might have positive effect on their adsorption by 270 graphenes. Both 1112TeCA and 112TCA were expected to have stronger van der Waals 271 interactions with graphenes than PCE and TCE, respectively due to their higher polarizabilities, 272 which would enhance their adsorption on graphenes. However, after solubility normalization, 273 1112TeCA and 112TCA did not show higher adsorption affinities to graphenes as compared to

274 PCE and TCE, which therefore indicated that the $\pi$-electrons of PCE and TCE possibly 275 contributed to their adsorption on graphenes through $\pi-\pi$ EDA complex to counterbalance the 276 contribution of van der Waals interactions to 1112TeCA and 112TCA adsorption. In addition, 277 their solubility-normalized adsorption isotherms once again demonstrated hydrophobic 278 interactions were dominant contributor for the adsorption of aliphatic SOCs on graphenes.

\subsection{The effect of background water components on adsorption of aliphatics}

\subsubsection{The effect of NOM}

To investigate the effect of NOM on adsorption of aliphatic SOCs by graphenes and compare NOM effects on adsorption capacities of GNS, rGO and SWCNT, adsorption of six

284 SOCs (PCE, TCE, 11DCE, 112TCA, 1112TeCA, and DBCP) with different properties by GNS, 285 rGO and SWCNT were tested in NOM solution. Their adsorption isotherms in DDW and NOM

286 solutions are presented in Figure 6. Freundlich isotherm parameters in DDI and NOM solution 287 are provided in Table S6 and S7 in Supporting Information, respectively. Two parameters, $\mathrm{R}_{\mu}$ 288 and $\mathrm{R}_{\mathrm{m}}$, as the ratios of $\mathrm{K}_{\mathrm{F} \mu}$ and $\mathrm{K}_{\mathrm{Fm}}$ in NOM preloading conditions to those in DDW, 289 respectively, were calculated to quantify the effect of NOM on the adsorption of the six aliphatic 
290

291

SOCs by different adsorbents. The lower $R_{\mu}$ and $R_{m}$ values indicate a greater reduction of adsorption capacity due to NOM preloading.

For GNS and rGO, the NOM effect on adsorption capacity of GNS were stronger than rGO, as indicated by lower $\mathrm{R}_{\mathrm{m}}$ values of GNS; and $\mathrm{rGO}$ exhibited comparable adsorption capacity in the presence of NOM and in DDW as reflected by $R_{m}$ values of rGO for the six SOCs ranging from 0.72 to 0.91 , which can be attributed to electrostatic repulsion of negatively charged NOM molecules and rGO surface. Because the low $\mathrm{pH}_{\mathrm{PZC}}$ value of rGO (Table 1), it had a net negative charge under neutral $\mathrm{pH}$ conditions; and NOM molecules generally carry a net negative charge at the same $\mathrm{pH}$, which would result in less NOM coating on the rGO surface. Furthermore, adsorption of six SOCs by GNS and rGO under NOM preloading conditions was compared to SWCNT adsorption. As shown in Table S7, $\mathrm{R}_{\mathrm{m}}$ values of SWCNT for all six SOCs were smaller than those of rGO and GNS, indicating that preloaded NOM exhibited stronger suppression on the adsorption of the aliphatic SOCs by SWCNT. This was attributed to more hindrance of the aliphatic SOC access to the adsorption sites on SWCNT due to the microporous structure of SWCNT bundles in water, in contrast to the flat sheet structure of GNS and rGO. These findings were similar to those of aromatic organic contaminant adsorption, where preloaded NOM exhibited much smaller impact on adsorption capacity of rGO than that of SWCNT [Apul et al., 2013]. However, although preloaded NOM exerted smaller effects on the adsorption capacities of GNS and rGO as compared to SWCNT, graphenes did not exhibit a major advantage, in terms of adsorption capacities, over SWCNT for the adsorption of aliphatic SOCs in the NOM solution (Figure S2).

The uptakes of all six SOCs decreased under NOM preloading conditions as reflected by $R_{m}$ values. However, there were no clear trends observed in $R_{m}$ values for each SOC; and the $R_{m}$ 
313 values did not correlate with the SOCs properties such as solubility, molecular size or

314 polarizability. In fact, preloaded NOM did not exert significantly different effects on the six

315 SOCs adsorption in terms of the $\mathrm{R}_{\mathrm{m}}$ values. Therefore, for the six aliphatic SOCs, their different

316 physicochemical properties did not cause large differences in NOM effects on their adsorption.

\subsubsection{The effect of $\mathrm{pH}$ and ionic strength}

Three aliphatic SOCs $\left(\mathrm{CCl}_{4}, \mathrm{TCE}\right.$, and $\left.\mathrm{DBCP}\right)$ with different molecular structure, molecular size and chain lengths were employed as probe molecules to explore the effect of

321 solution $\mathrm{pH}$ on adsorption of aliphatic SOCs by graphenes. The adsorption isotherms of $\mathrm{CCl}_{4}$,

322 TCE and DBCP on GNS and rGO under different $\mathrm{pH}$ conditions are presented in Figure S3 in

323 the Supporting Information. The change in $\mathrm{pH}$ did not influence the adsorption of these three 324 aliphatic SOCs on GNS and rGO (except for DBCP under pH 11 conditions). Since the tested 325 compounds are non-ionic, their adsorption was independent from solution $\mathrm{pH}$. However, as $\mathrm{pH}$ 326 increased, the dissociation of oxygen-containing functional groups on graphene surfaces 327 (especially rGO) also did not show any influence indicating that adsorption of these three 328 aliphatic SOCs occurred on the hydrophobic sites of graphene surface where there were no 329 oxygen-containing functional groups. For DBCP at pH 11, we observed a higher adsorption by 330 graphenes, but actually this does not reflect its real adsorption affinity. Because degradation and 331 transformation of DBCP at high $\mathrm{pH}$ might occur during the adsorption experiments indicated by

332 the very low ratio (0.10-0.15) between measured blank concentrations and calculated ones.

To investigate the effect of ionic strength, the same aliphatic SOCs selected for $\mathrm{pH}$ experiments were used in the adsorption experiments. Two potential impacts can be observed:

335 (1) increasing ionic strength enhances the activity coefficient of hydrophobic organic 
336 compounds, leading to a decrease in their solubility (i.e. salting out effect), which is favorable

337 for SOCs adsorption [Zhang et al., 2010]; and (2) the ions may penetrate into the diffuse double

338 layer surrounding the graphene surfaces and eliminate the repulsive energy between the

339 adsorbents, facilitating the formation of a more compact aggregation structure (i.e. squeezing-

340 out), which is unfavorable for SOCs adsorption [Zhang et al., 2010]. Increasing ionic strength

341 had negligible effect on adsorption of the aliphatic SOCs by both GNS and rGO (Figure S3).

342 Therefore, the results indicated that either the contribution of salting-out effect to the adsorption

343 of these aliphatic SOCs was equivalent to that of the squeezing-out effect or both the salting-out

344 effect and squeezing-out effect were too weak to influence the adsorption of the SOCs on 345 graphenes.

347 4. Conclusions

Graphene nanomaterials adsorbed halogenated aliphatic SOCs, however the overall 349 adsorption capacities of graphene nanomaterials were smaller than those of carbon nanotubes 350 and activated carbon in the presence of NOM. Hydrophobicity was the dominant contributor for 351 the adsorption of aliphatic SOCs on graphenes, but it was not the only mechanism controlling the 352 adsorption, $\pi-\pi$ EDA interaction and van der Waals interaction appear to be the additional 353 mechanisms contributing to the adsorption of aliphatic SOCs on graphenes. It should be noted that 354 these adsorption interactions were observed for the selected halogenated aliphatic contaminants with 355 comparable molecular configuration and sizes in this study. Further research is warranted to confirm and 356 extend the findings to larger and more complex aliphatic molecules or different classes of contaminants.

357 Among the three background water characteristics examined, NOM showed the most important 358 effect on adsorption, whereas the changes in $\mathrm{pH}$ and ionic strength had a negligible effect on the 
359 adsorption of selected halogenated aliphatic SOCs. NOM preloading exerted minimal effect on 360 adsorption capacities of rGO for aliphatic SOCs, which was attributed to low degree of NOM 361 coating on rGO surface due to electrostatic repulsion between negatively charged NOM 362 molecules and rGO. NOM exhibited severe suppression on adsorption capacities of SWCNT

363 than those of graphenes. This was attributed to microporous structure of SWCNT aggregates as 364 compared with flat sheet structure of graphenes. In terms of adsorption capacities, graphenes did 365 not exhibit a major advantage over SWCNT and HD3000 for the adsorption of aliphatic SOCs. 366 Future studies also need to examine adsorption kinetics to assess whether graphenes exhibit 367 advantages over other carbonaceous adsorbents for halogenated aliphatic SOCs.

\section{Acknowledgement}

This work was supported by a research grant from the National Science Foundation

371 (CBET 0967425). However the manuscript has not been subjected to the peer and policy review 372 of the agency and therefore does not necessarily reflect it views. 


\section{References}

375

376

377

378

379

380

381

382

383

384

385

386

387

388

389

390

391

392

393

394

395

396

397

398

399

400

401
Apul, O.G., Wang, Q.L., Zhou, Y., Karanfil, T. 2013. Adsorption of aromatic organic contaminants by graphene nanosheets: comparison with carbon nanotubes and activated carbon. Water Research 47 (4), 1648-1654.

Carter, M.C., Kildfuff, J.E., Weber Jr, W.J. 1995. Site energy distribution analysis of preloaded adsorbent. Environmental Science and Technology 29 (7), 1773-1780.

Chen, J., Chen, W., Zhu, D. 2008. Adsorption of nonionic aromatic compounds to single-walled carbon nanotubes: Effects of aqueous solution chemistry. Environmental Science and Technology 42 (19), 7225-7230.

Dastgheib, S.A., Karanfil, T., Cheng, W. 2004. Tailoring activated carbons for enhanced removal of natural organic matter from natural waters. Carbon 42 (3), 547-557.

Fontecha-Camara, M.A., Lopez-Ramon, M.V., Alvarez-Merine, M.A., Morene-Castilla, C. 2007. Effect of surface chemistry, solution $\mathrm{pH}$, and ionic strength on the removal of herbicides diuron and amitrole from water by an activated cabon fiber. Langmuir 23 (3), 1242-1247.

Gao, Y., Li, Y., Zhang, L., Huang, H., Hu, J.J., Shah, S.M., Su, X.G. 2012. Adsorption and removal of tetracycline antibiotics from aqueous solution by graphene oxide. Journal of Colloid and Interface Science 368 (1), 540-546.

Geim, A.K., 2009. Graphene: status and prospects. Science 324 (5934), 1530-1534.

Geim, A.K., Novoselov, K.S. 2007. The rise of graphene. Nature Materials 6 (3), 183-191. 
406

407

408

409

410

411

412

413

414

415

416

417

418

419

420

421

422

423

424

425

426

427

428

429

430

431

Giles, R.G.F., Green, I.R., Oosthuizen, F.J., Taylor, C.P. 2003. Syntheses of asymmetric 2benzopyrans. The influence of aromatic halogen substituents on the intramolecular cyclisation of enantiopure tethered phenolic lactaldehydes. Archive for Organic Chemistry 2002 (9), 99-116.

Ji, L.L., Chen, W., Xu, Z.Y., Zheng, S.R., Zhu, D.Q. 2013. Graphene Nanosheets and graphite oxide as promising adsorbents for removal of organic contaminants from aqueous solution. Journal of Environmental Quality 42 (1), 191-198.

Karanfil, T., Erdogan, I., Schlautman, M.A. 2003. Selecting filter membranes for measuring DOC and UV254. Journal of American Water Works Association, 95, (3) 86-100.

Karanfil, T., Dastgheib, S.A. 2004. Trichloroethylene adsorption by fibrous and granular activated carbons: Aqueous phase, gas phase and water vapor adsorption studies. Environmental Science and Technology 38 (22), 5834-5841.

Lee, C., Wei, X., Kysar, J.W., Hone, J. 2008. Measurement of the elastic properties and intrinsic strength of monolayer graphene. Science 321 (5887), 385-388.

Li, D., Kaner, R.B., 2008. Graphene-based materials. Science 320 (5880), 1170-1171.

Li, L., Quinlivan, P.A., Knappe, D.R.U.] 2002. Effects of activated carbon surface chemistry and pore structure on the adsorption of organic contaminants from aqueous solution. Carbon 40 (12), 2085-2100.

Li, Y.H., Du, Q.J., Liu, T.H., Peng, X.J., Wang, J.J., Sun, J.K., Wang, Y.H., Wu, S.L., Wang, Z.H., Xia, Y.Z., Xia, L.H.] 2013. Comparative Study of methylene blue dye adsorption onto activated carbon, graphene oxide, and carbon nanotubes. Chemical Engineering Research and Design 91 (2), 361-368. 
451

452

453

454

455

456

457

458

459

460

461

462

Li, Y.H., Du, Q.J., Liu, T.H., Sun, J.K., Jiao, Y.Q., Xia, Y.Z., Xia, L.H., Wang, Z.H., Zhang, W., Wang, K.L., Zhu, H.W., Wu, D.H. 2012. Equilibrium, kinetic and thermodynamic studies on the adsorption of phenol onto graphene. Materials Research Bulletin 47 (8), 18981904.

Lin, D., Xing, B. 2008. Adsorption of phenolic compounds by carbon nanotubes: Role of aromaticity and substitution of hydroxyl groups. Environmental Science and Technology 42 (19), 7254-7259.

Lu, C.S., Chung, Y.L., Chang, K.F. 2005. Adsorption of trihalomethanes from water with carbon nanotubes. Water Research 39 (6), 1183-1189.

Novoselov, K.S., Geim, A.K., Morozov, S.V., Jiang, D., Zhang, Y., Dubonos, S.V., Grigorieva, I.V., Firsov, A.A. 2004. Electric field effect in atomically thin carbon films. Science 306 (5696), 666-669.

Novoselov, K.S., Jiang, D., Schedin, F., Booth, T.J., Khotkevich, V.V., Morozov, S.V., Geim, A.K. 2005. Two-dimensional atomic crystals. Proceedings of the National Academy of Sciences 102 (30), 10451-10453.

Nowack, B., Ranville, J.F., Diamodn, S., Gellego-Urrea, J.A., Metcalfe, C., Rose, J., Horne, N., Koelmans, A.A., Klaine, S.J. 2012. Potential scenarios for nanomaterial release and subsequent alteration in the environment. Environmental Toxicology and Chemistry 31 (1), 50-59.

Ramesha, G.K., Vijaya Kumara, A., Muralidhara, H.B., Sampath, S. 2011. Graphene and graphene oxide as effective adsorbents toward anionic and cationic dyes. Journal of Colloid and Interface Science 361 (1), 270-277.

Robin, G. F. Giles.; Ivan, R. Green.; Francois, J. Oosthuizen.; C. Peter Taylor. 2002. Syntheses of asymmetric 2-benzopyrans. The influence of aromatic halogen substituents on the 
481

482

483

484

485

486

487

488

489

490

491

492

493

intramolecular cyclisation of enantiopure tethered phenolic lactaldehydes. Archive for Organic Chemistry (9), 99-116.

Sharma, P., Das, M.R. 2013. Removal of a cationic dye from aqueous solution using graphene oxide nanosheets: investigation of adsorption parameters. Chemical Engineering Journal 58 (1), 151-158.

Song, H., Orr, O., Hong, Y., Karanfil, T. 2009. Isolation and fractionation of natural organic matter: evaluation of reverse osmosis performance and impact of fractionation parameters. Environmental Monitoring and Assessment 153 (1), 307-321.

Stoller, M.D., Park, S.J., Zhu, Y.,W., An, J.,H., Ruoff, R.S. 2008. Graphene-based ultracapacitors. Nano Letters 8 (10), 3498-3502.

Upadhyayula, V.K.K., Deng, S., Mitchell, M.C., Smith, G.B. 2009. Application of carbon nanotube technology for removal of contaminants in drinking water: A review. Science of the Total Environment 408 (1), 1-13.

Wu, T., Cai, X., Tan, S.Z., Li, H.Y., Liu, J.S., Yang, W.D. 2011. Adsorption characteristics of acrylonitrile, P-toluenesulfonic acid, 1-naphthalenesulfonic acid and methylene blue on graphene in aqueous solutions. Chemical Engineering Journal 173 (1), 144-149.

Yang, K., Wu, W.H., Jing, Q.F., Zhu, L.Z. 2008. Aqueous adsorption of aniline phenol, and their substitutes by multi-walled carbon nanotubes. Environmental Science and Technology 42 (21), 7931-7936.

Yang, K., Xing, B. 2009. Adsorption of fulvic acid by carbon nanotubes from water. Environmental Pollution 157 (4), 1095-1100.

Yu, J.G., Zhao, X.H., Yang, H., Chen, X.H., Yang, Q., Yu, L.Y., Jiang, J.H., Chen, X.Q. 2014. Aqueous adsorption and removal of organic contaminants by carbon nanotubes. Science of the Total Environment 482-483, 241-251.

Yu, J.G., Yu, L.Y., Yang, H., Liu, Q., Chen, X.H., Jiang, X.Y., Chen, X.Q., Jiao, F.P. 2015. Graphene nanosheets as novel adsorbents in adsorption, preconcentration and removal of gases, organic compounds and metal ions. Science of the Total Environment 502, 70-79. 
498

499

500

501

502

503

504

505

506

507 508

509

510

511

512

513

514

515

516

517

518

Zhang, S., Shao, T., Kose, H.S., Karanfil, T. 2010. Adsorption of aromatic compounds by carbonaceous adsorbents: A comparative study on granular activated carbon, activated carbon fiber, and carbon nanotubes. Environmental Science and Technology 44 (16), 6377-6383.

Zhang, S.J., Shao, T., Bekaroglu, S.S.K., Karanfil, T. 2010. Adsorption of synthetic organic chemicals by carbon nanotubes: Effects of background solution chemistry. Water Research 44 (6), 2067-2074.

Zhang, Y., Ali, S.F., Dervishi, E., Xu, Y., Li, Z., Casciano, D., Biris, A.S. 2010. Cytotoxicity effects of graphene and single-wall carbon nanotubes in neural phaeochromocytomaderived PC12 cells. Journal of the American Chemical Society 4 (6), 3181-3186.

Zhao, G., Jiang, L., He, Y., Li, J., Dong, H., Wang, X., Hu, W. 2011. Sulfonated graphene for persistent aromatic pollutant management. Advanced Materials 23 (34), 3959-3963.

Zhao, G.X., Li, J.X., Wang, X.K. 2011. Kinetic and thermodynamic study of 1-naphthol adsorption from aqueous solution to sulfonated graphene nanosheets. Chemical Engineering Journal 173 (1), 185-190.

Zhao, J., Wang, Z.Y., White, J.C., Xing, B.S. 2014. Graphene in the aquatic environment: adsorption, dispersion, toxicity and transformation. Environmental Science and Technology 48 (17), 9995-10009. 
Table 1. Physicochemical properties of adsorbents

\begin{tabular}{|c|c|c|c|c|c|c|c|}
\hline \multirow[t]{2}{*}{ Adsorbent } & \multirow{2}{*}{$\begin{array}{c}\operatorname{SSA}_{\mathrm{BET}}{ }^{\mathrm{a}} \\
\mathbf{m}^{2} / \mathrm{g}\end{array}$} & \multirow{2}{*}{$\begin{array}{c}V_{T}^{b} \\
\mathbf{c m}^{3} / g\end{array}$} & \multicolumn{3}{|c|}{ DFT Pore Volume Distribution $^{c}$} & \multirow{2}{*}{$\begin{array}{c}\text { Oxygen Content } \\
\%\end{array}$} & \multirow{2}{*}{$\mathrm{pH}_{\mathrm{pzc}}$} \\
\hline & & & $\begin{array}{c}V_{\text {micro }}(<2 \mathrm{~nm}) \\
\mathrm{cm}^{3} / \mathrm{g},(\%)\end{array}$ & $\begin{array}{c}V_{\text {meso }}(2-50 \mathrm{~nm}) \\
\mathrm{cm}^{3} / \mathrm{g},(\%)\end{array}$ & $\begin{array}{c}V_{\text {macro }}(>50 \mathrm{~nm}) \\
\mathrm{cm}^{3} / \mathrm{g},(\%)\end{array}$ & & \\
\hline GNS & 666 & 3.138 & $0.065,(2.1)$ & 1.196, (38.1) & $1.877,(59.8)$ & 0.8 & 9.8 \\
\hline rGO & 497 & 0.530 & $0.081,(15.3)$ & 0.377, (71.1) & $0.072,(13.6)$ & 17.5 & 4.1 \\
\hline SWCNT & 537 & 1.240 & $0.117,(9.4)$ & 0.581, (46.9) & $0.542,(43.7)$ & 0.9 & 7.5 \\
\hline HD3000 & 642 & 0.775 & 0.108, (13.9) & 0.449, (57.9) & $0.218,(28.1)$ & 3.4 & 6.9 \\
\hline
\end{tabular}

$520{ }^{a}$ Specific surface area calculated with the Brunauer-Emmett-Teller (BET) model, ${ }^{\mathrm{b}}$ Total pore volume calculated from single point adsorption at P/P $\mathrm{P}_{0}=0.99,{ }^{\mathrm{c}}$ Pore

521 volume in each pore size range obtained from the density functional theory (DFT) analysis. 
Table 2. Selected properties of aliphatic SOCs

\begin{tabular}{|c|c|c|c|c|c|c|c|}
\hline SOC & Abbreviation & $\begin{array}{l}\mathrm{MW}^{\mathrm{a}} \\
\mathrm{g} / \mathrm{mol}\end{array}$ & $\begin{array}{c}\text { Density } \\
\text { g/cm }\end{array}$ & $\begin{array}{c}\mathrm{MV}^{\mathrm{b}} \\
\mathrm{cm}^{3} / \mathrm{mol}\end{array}$ & $\begin{array}{c}\mathrm{C}_{\mathrm{S}}^{\mathrm{c}} \\
\mathrm{mg} / \mathrm{L}\end{array}$ & $\operatorname{LogK}_{\text {ow }}{ }^{d}$ & Polarizability $^{\mathrm{e}}$ \\
\hline trichloroethylene & TCE & 131 & 1.46 & 89.7 & 1183 & 2.42 & 0.37 \\
\hline tetrachloroethylene & PCE & 166 & 1.62 & 102.5 & 224 & 3.40 & 0.44 \\
\hline 1,1,1-trichloroethane & 111TCA & 133 & 1.32 & 100.8 & 1358 & 2.49 & 0.41 \\
\hline 1,1,2-trichloroethane & 112TCA & 133 & 1.44 & 92.4 & 4483 & 1.89 & 0.68 \\
\hline carbon tetrachloride & $\mathrm{CCl}_{4}$ & 154 & 1.59 & 96.9 & 790 & 2.83 & 0.38 \\
\hline 1,1-dichloroethylene & 11DCE & 97 & 1.21 & 80.2 & 2375 & 1.32 & 0.34 \\
\hline 1,2-dichloropropane & 12DCP & 113 & 1.16 & 97.4 & 2819 & 2.28 & 0.68 \\
\hline 1,2-dibromoethane & 12DBE & 188 & 2.17 & 86.6 & 4177 & 1.96 & 0.76 \\
\hline 1,1,1,2-tetrachloroethane & 1112ТеCA & 168 & 1.55 & 108.3 & 1103 & 2.93 & 0.63 \\
\hline 1,2-dibromo-3-chloropropane & DBCP & 236 & 2.08 & 113.5 & 985 & 2.43 & 0.78 \\
\hline
\end{tabular}

$523 \quad{ }^{\mathrm{a}}$ molecular weight; ${ }^{\mathrm{b}}$ molar volume; ${ }^{\mathrm{c}}$ water solubility at $25{ }^{\circ} \mathrm{C}$ obtained from the Material Safety Data Sheet of each compound; ${ }^{\mathrm{d}}$ octanol-water partitioning coefficient

524 simulated with ACDLABS11.0 (ChemSketch and ACD/3D Viewer); ${ }^{\mathrm{e}}$ polarizability obtained from ACD/ADME Suite 5.0. 

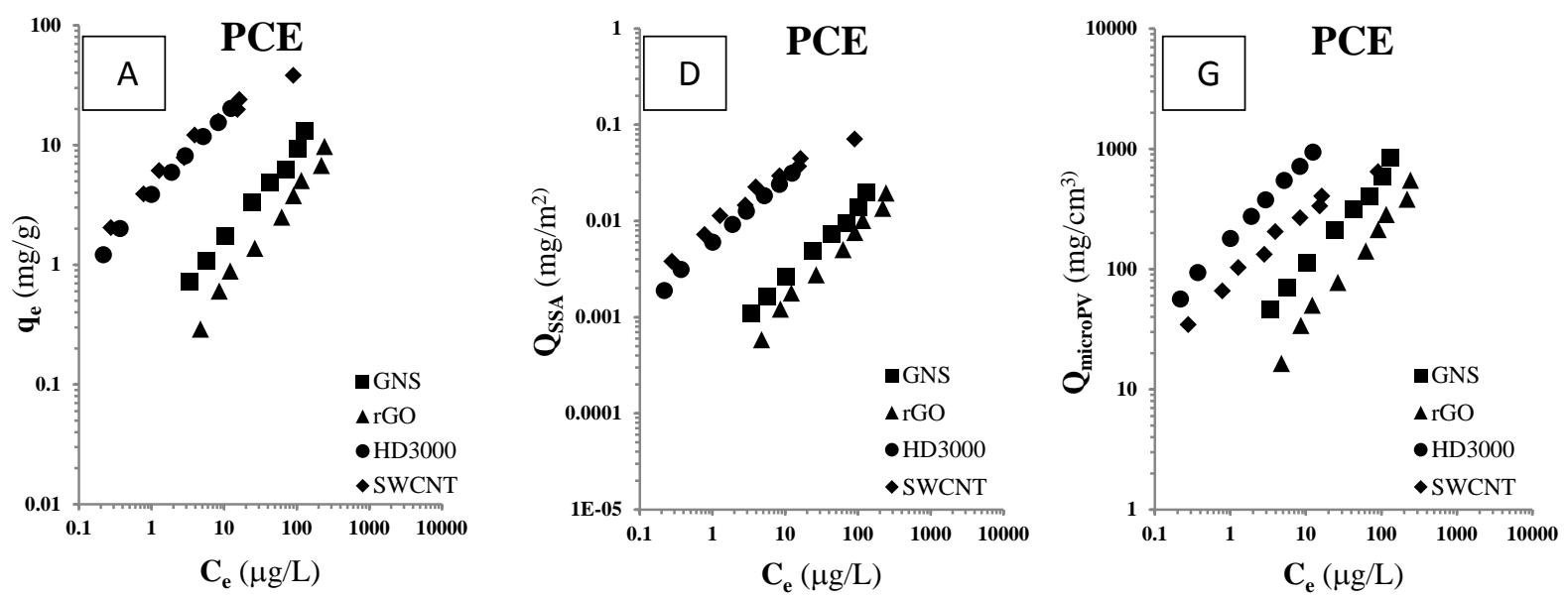

528
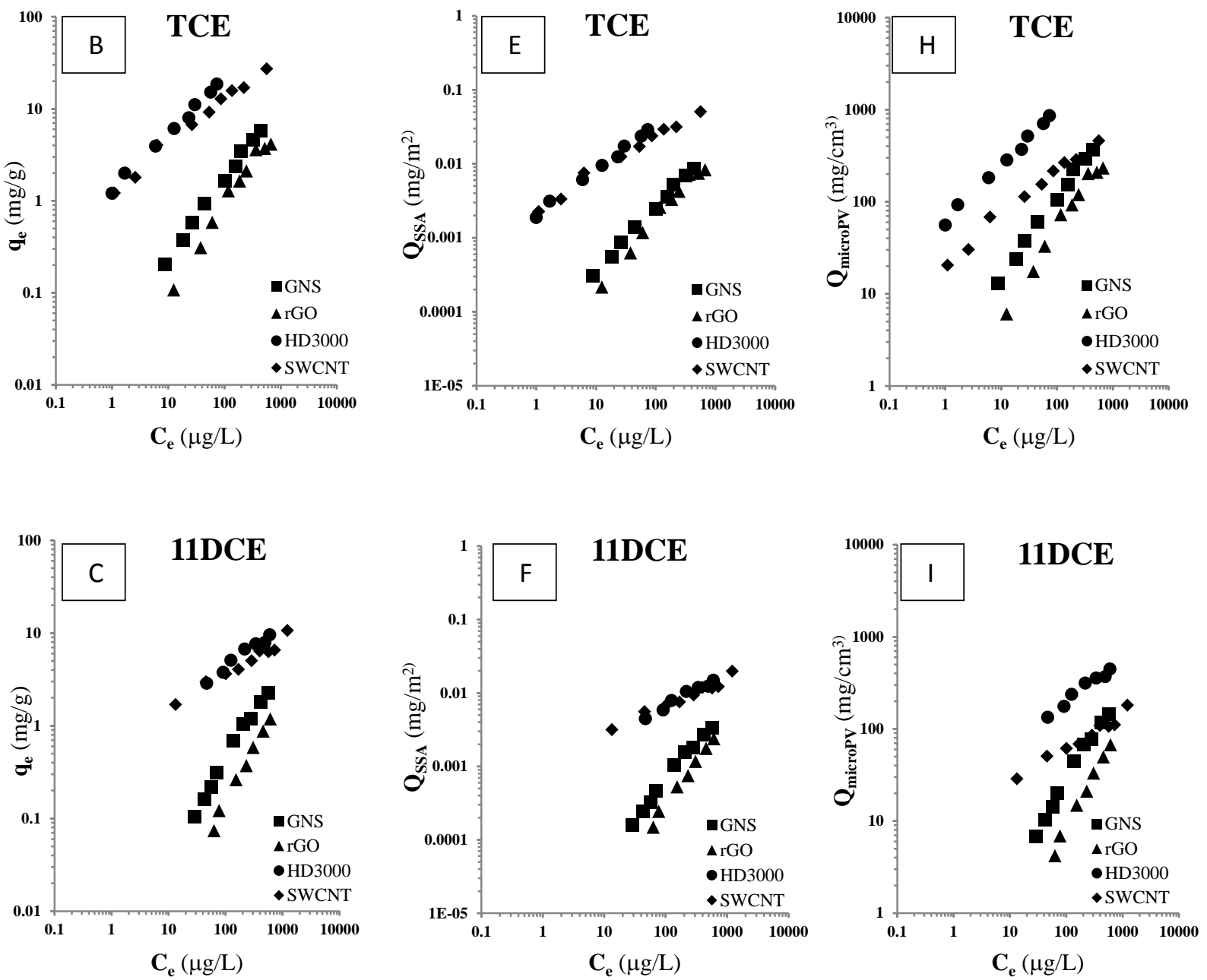

Figure 1. Adsorption isotherms in DDW (A) PCE mass basis, (B) TCE mass basis, (C) 11DCE mass basis, (D) PCE surface area normalized, (E) TCE surface area normalized, (F) 11DCE surface area normalized, (G) PCE micropore volume normalized, (H) TCE micropore volume normalized, and (I) 

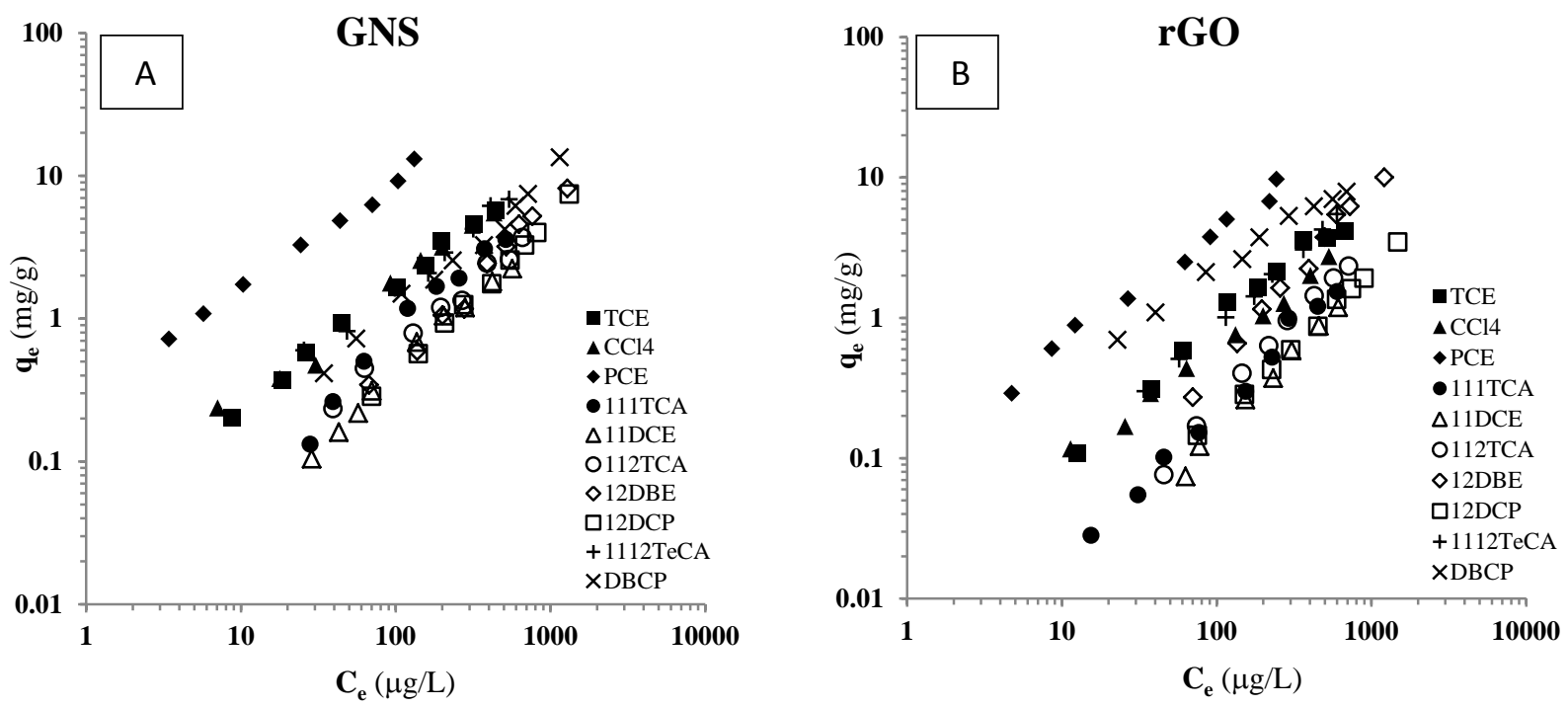

537
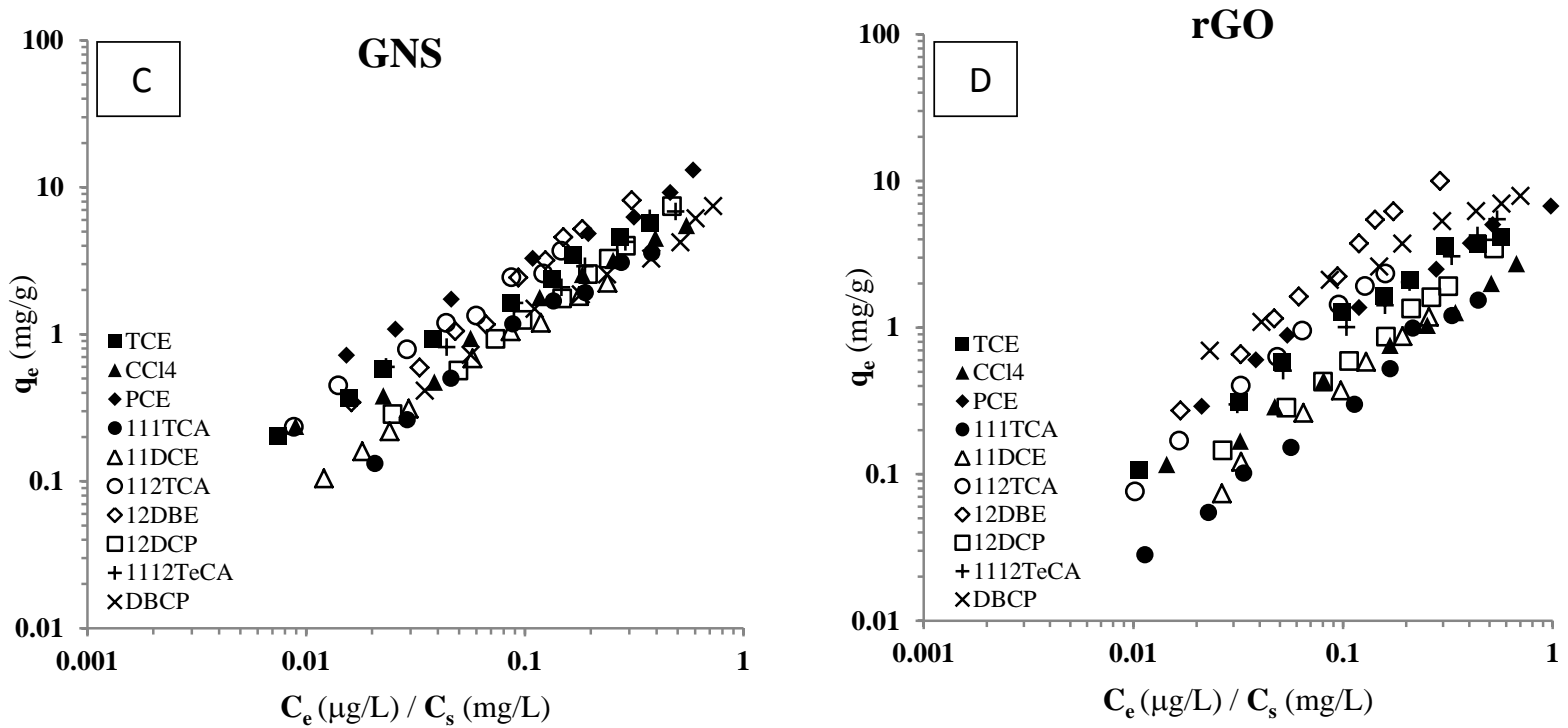

Figure 2. Adsorption isotherms of ten aliphatic SOCs by GNS and rGO in DDW (A) GNS mass basis, (B) rGO mass basis, (C) GNS solubility normalized, (D) rGO solubility normalized . 

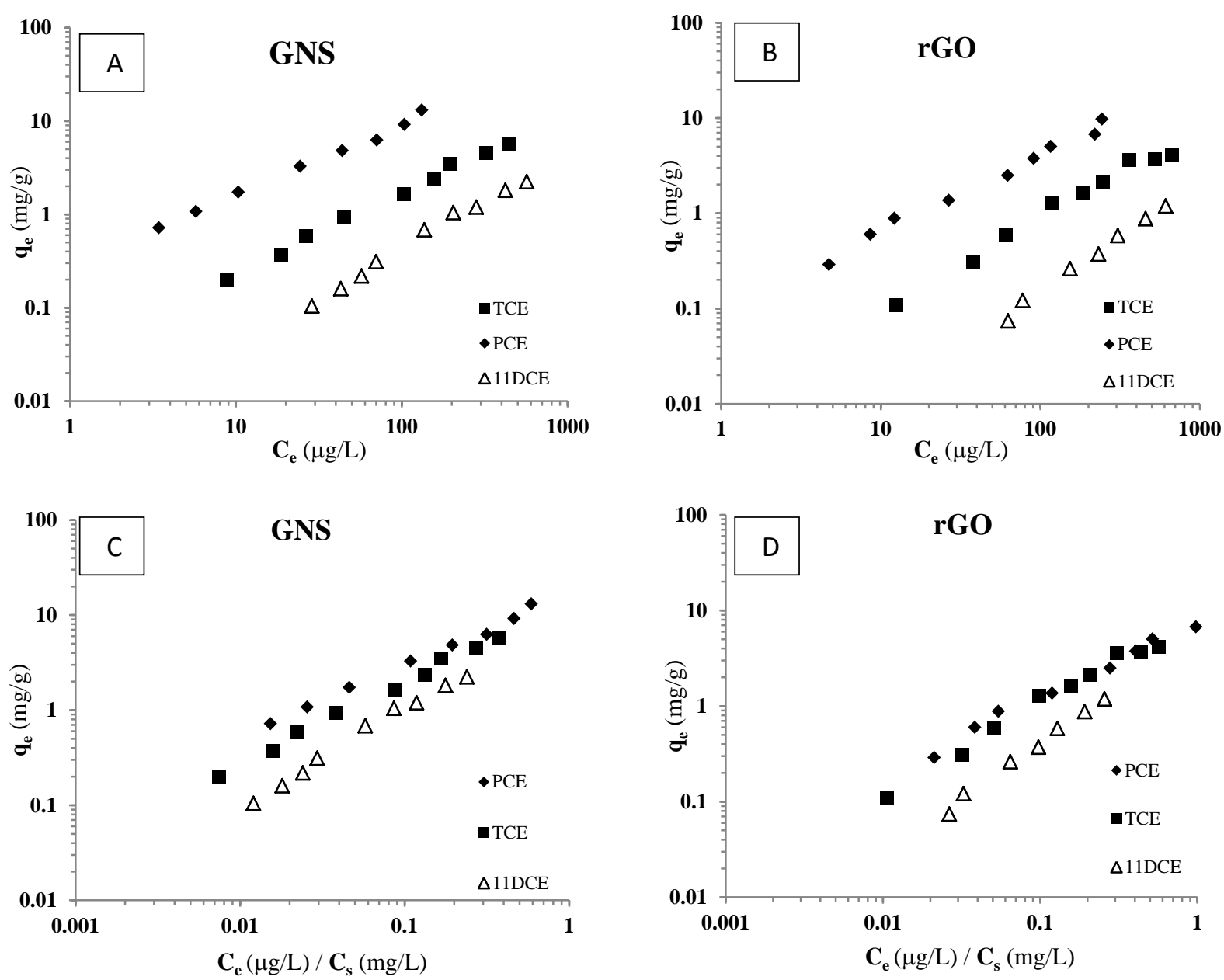

544
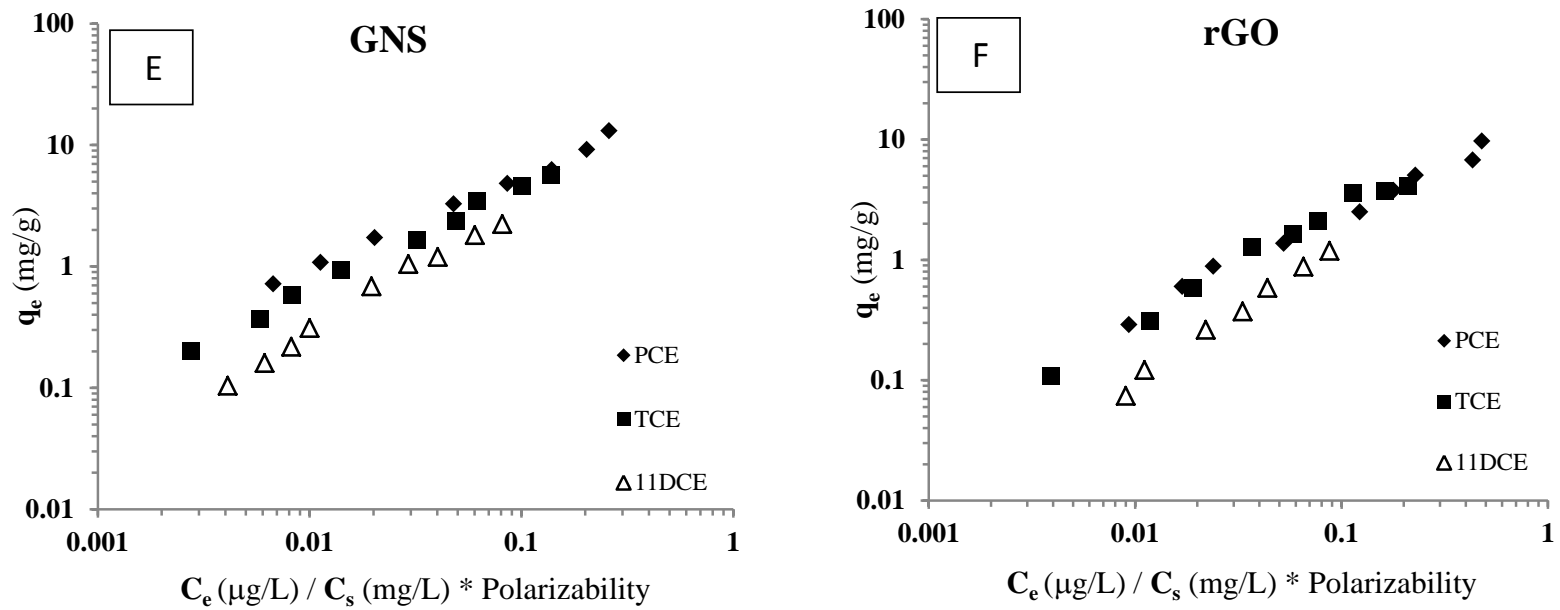

Figure 3. Adsorption isotherms of PCE, TCE and 11DCE in DDW (A) GNS mass basis, (B) rGO mass basis, (C) GNS solubility normalized, (D) rGO solubility normalized, (E) GNS polarizability normalized, and (F) rGO polarizability normalized. 


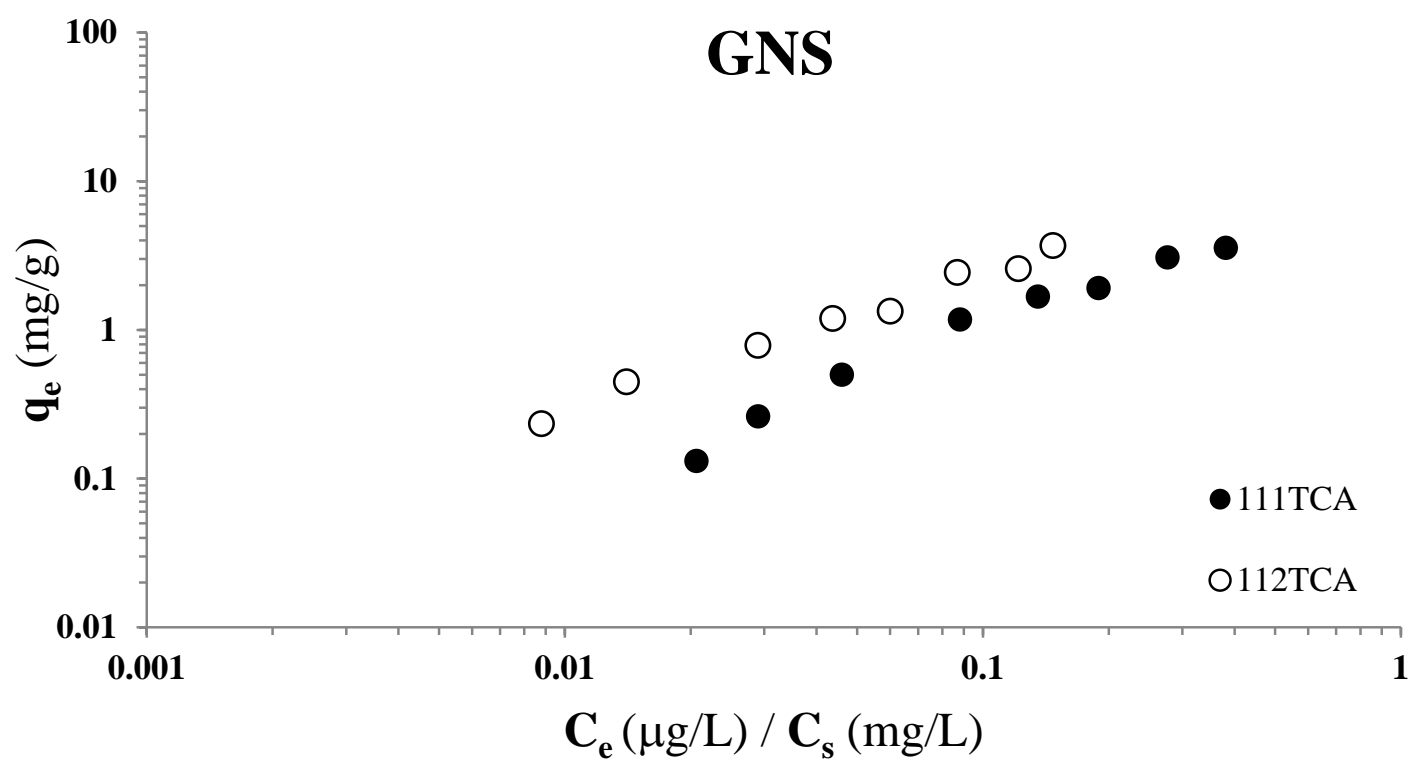

551

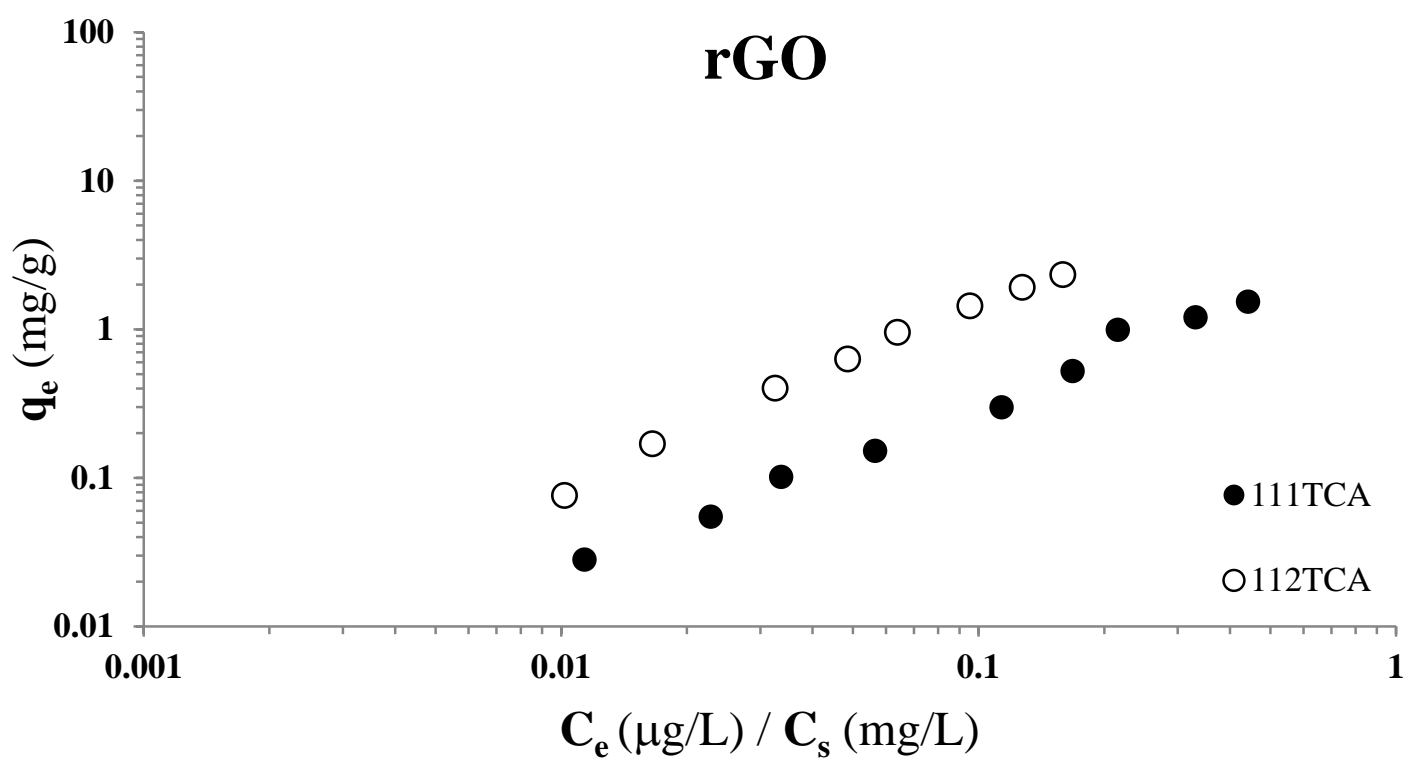

553 Figure 4. Comparison of 111TCA and 112TCA solubility-normalized adsorption isotherms on GNS and 554 rGO in DDW.

555

556

557 

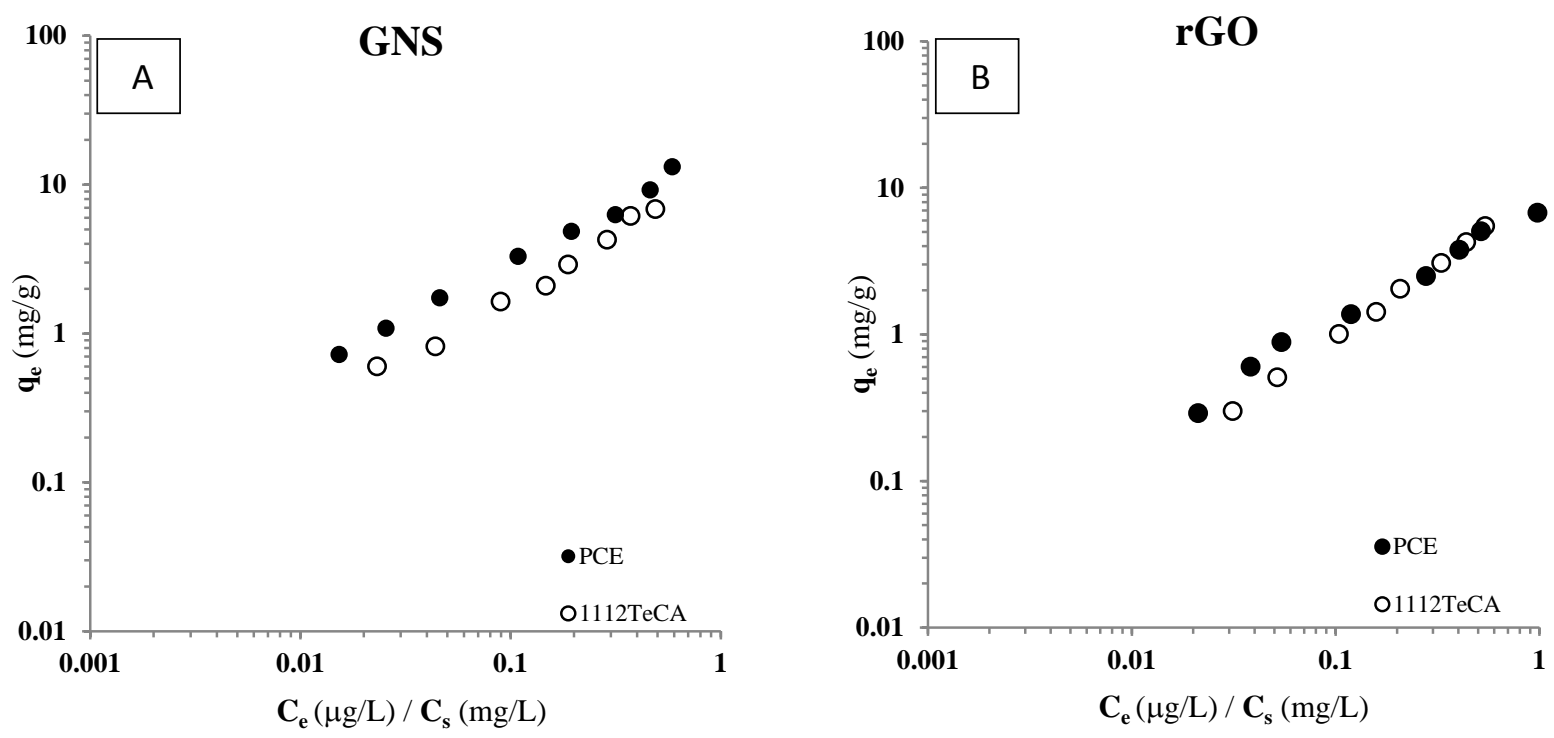

558

559
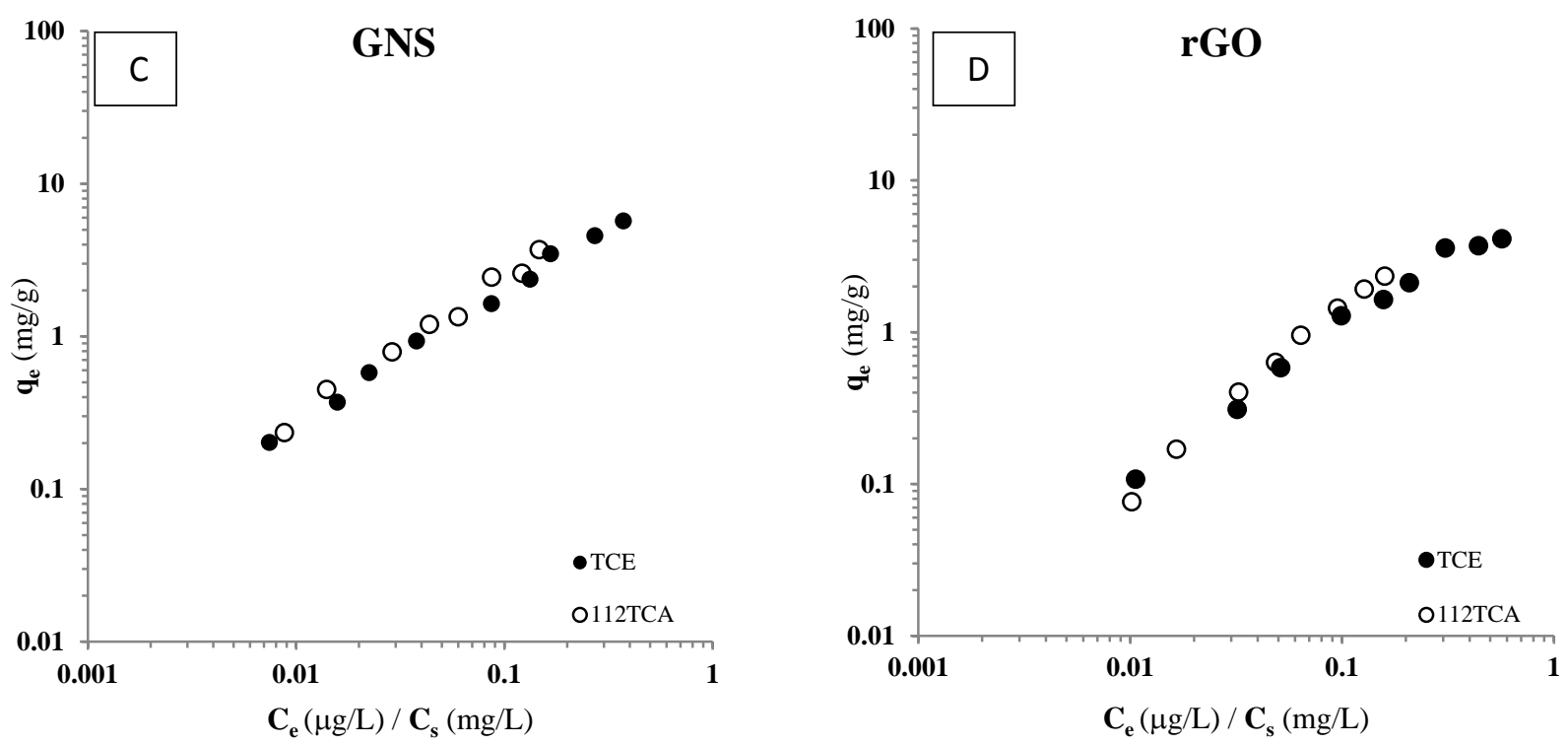
561 Figure 5. Comparison of PCE vs. 1112TeCA and TCE vs. 112TCA solubility normalized adsorption 563 TCE vs. 112TCA-GNS, and (D) TCE vs. 112TCA- rGO. 

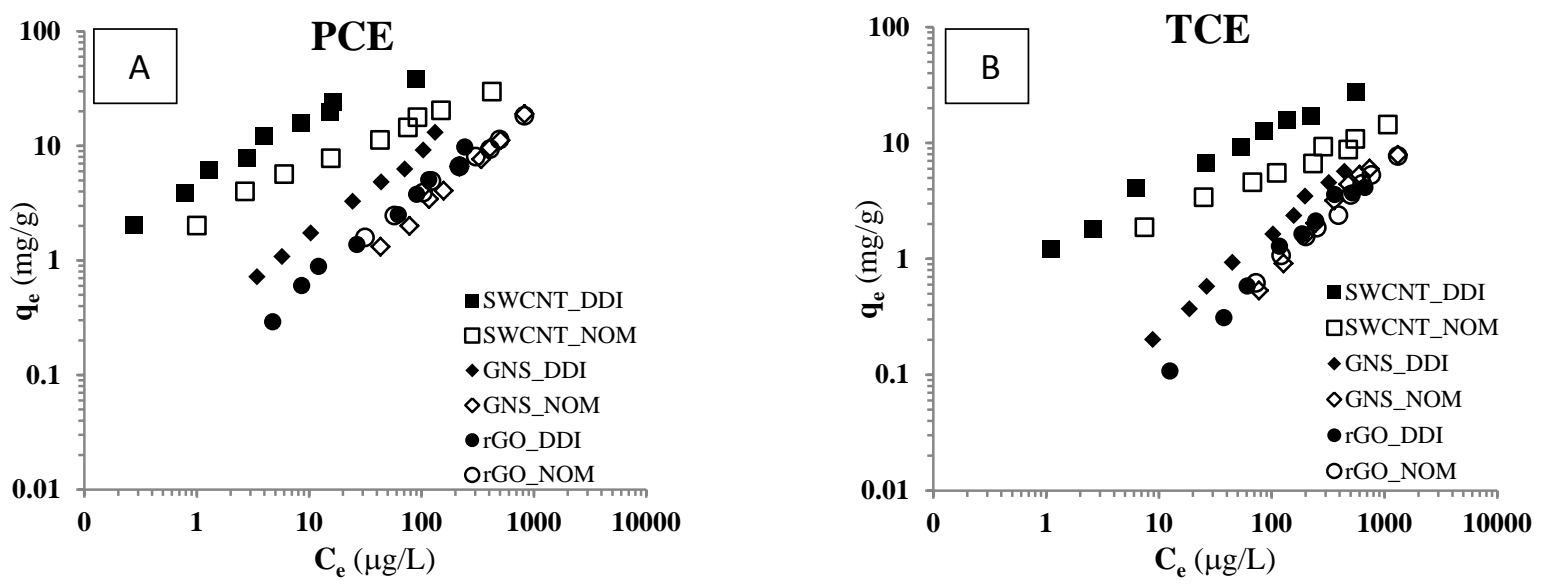

565
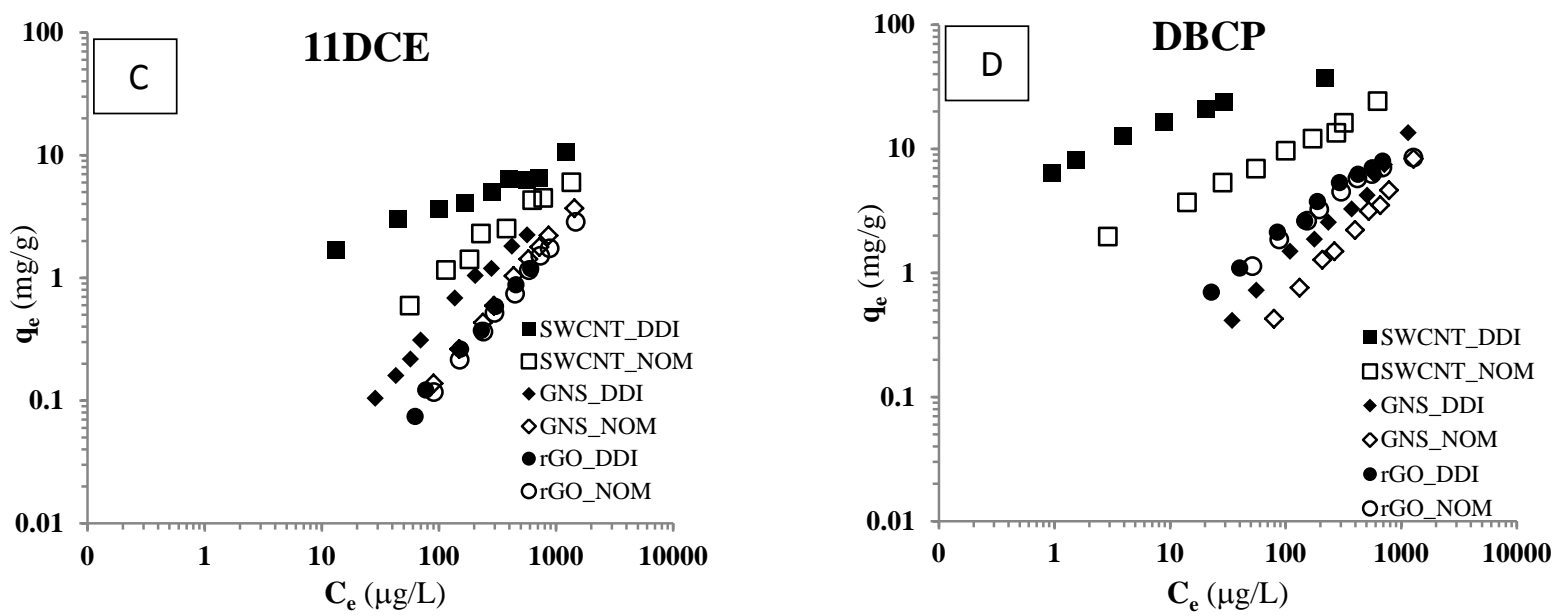

567
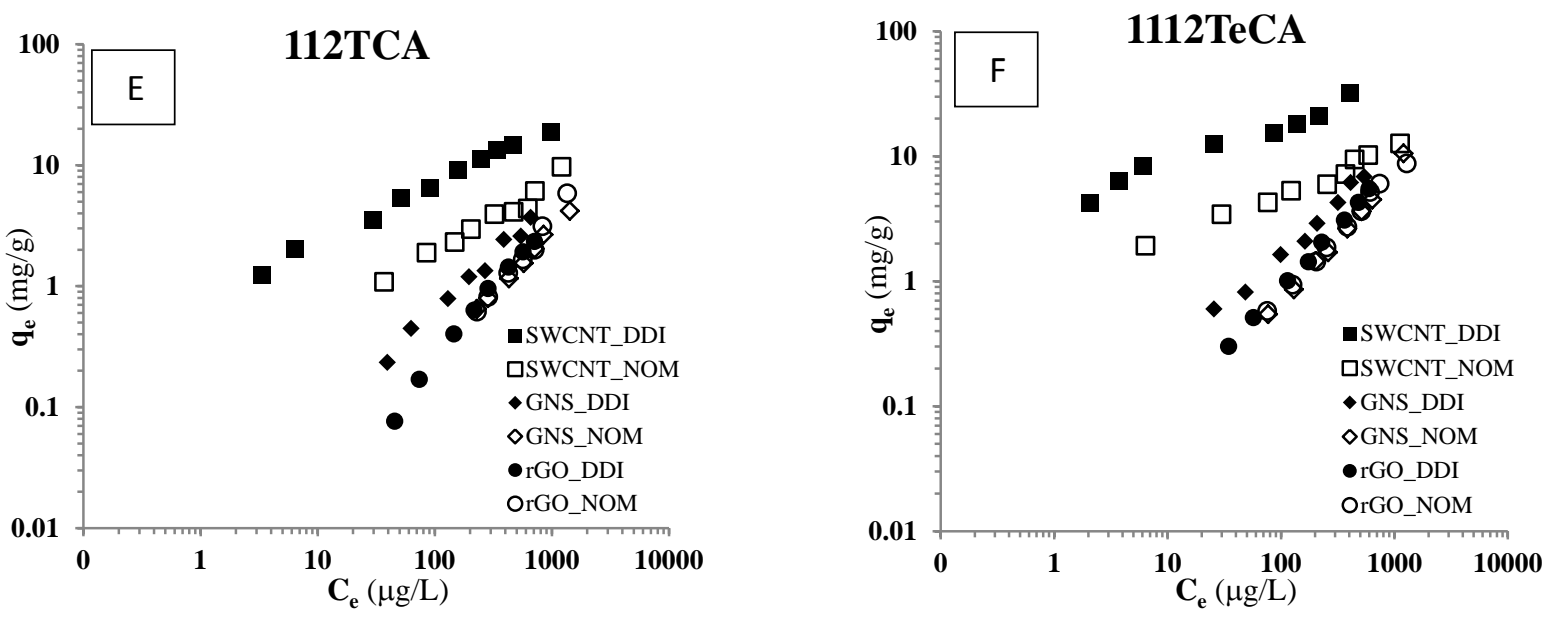

569 Figure 6. Adsorption isotherms in DDW and under NOM preloading conditions (A) PCE, (B) TCE, (C) 
Graphical Abstract (for review)

TOC Art

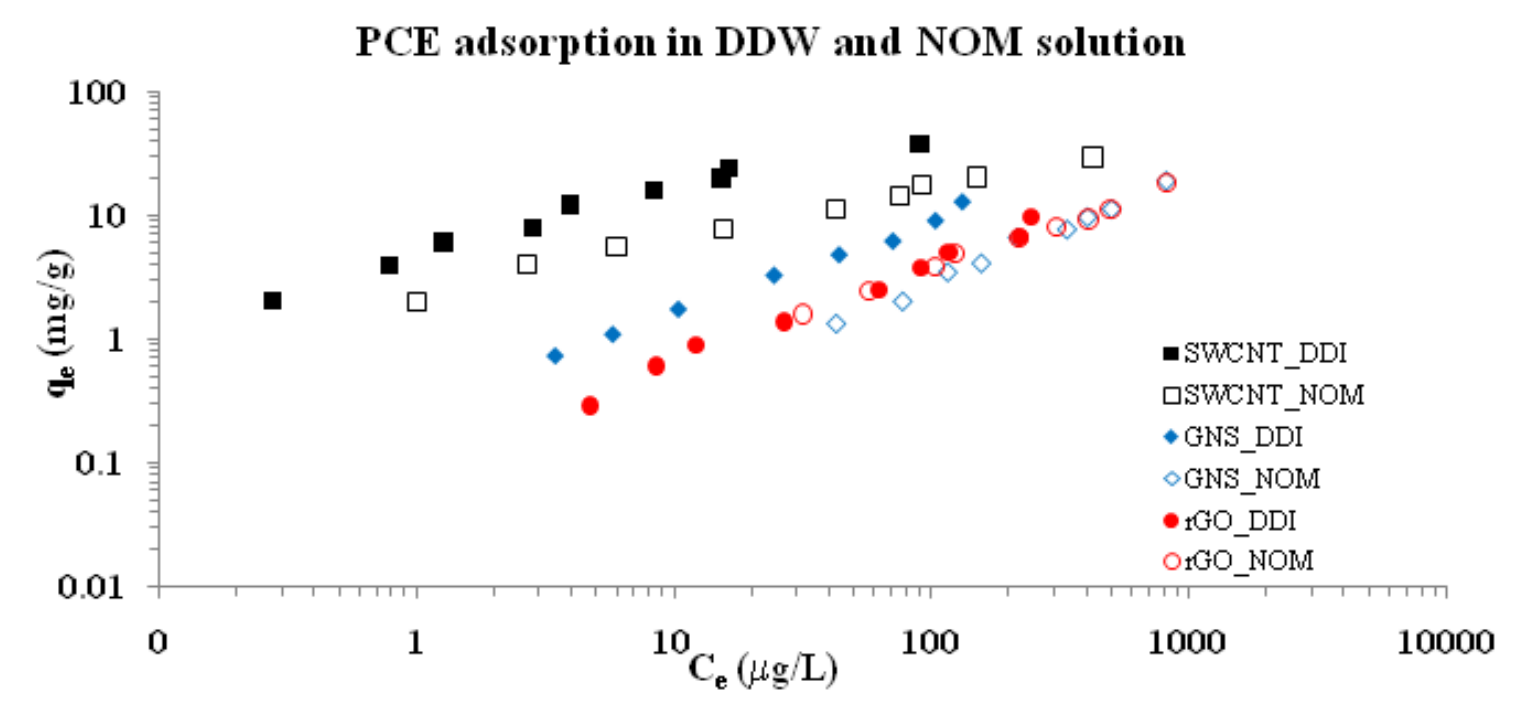

